\title{
FUNCTION GENERATOR TO STUDY THE DIELECTRIC BREAKDOWN IN THIN FILM STRUCTURES
}

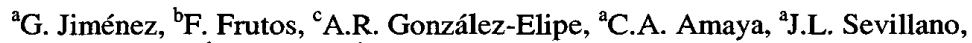 \\ ${ }^{\mathrm{b}} \mathrm{M}$. Acedo, ${ }^{\mathrm{b}}$ A. Jiménez and ${ }^{\mathrm{a}} \mathrm{F}$. Díaz del Río \\ ${ }^{a}$ Departamento de Arquitectura y Tecnología de Computadores, Fac. Informática, Univ. de Sevilla, Avda. Reina \\ Mercedes s/n, 41012-Sevilla, Spain. \\ ${ }^{b}$ Departamento de Física Aplicada I, Fac. Informática, Univ. de Sevilla, Avda. Reina Mercedes s/n, 41012, Spain. \\ ${ }^{c}$ Instituto de Ciencia de Materiales de Sevilla (CSIC-Univ. de Sevilla). Avda. Américo Vespucio s/n. 41092 Sevilla, \\ Spain.
}

\section{INTRODUCTION}

Characterization of breakdown in thin films requires testing experimental samples with different voltage patterns in order to obtain dielectric parameters, e.g. breakdown voltage and charge to breakdown. The most common voltage patterns used are voltage ramp and step [1]. This paper describes design and implementation of a function generator especially suited for thin films testing. The generator can be controlled using a computer, performing many different voltage patterns. This feature makes it a very versatile instrument, useful in a wide range of experiments. The system is based on a switching DC power supply (Flyback converter) [2], controlled by a computer (on-board microcontroller). The control system is a PD with a feedforward loop [3].

Output voltage waveform is generated by the microcontroller using a stored data pattern, with a sampling interval of $0.1 \mathrm{~ms}$. Stored data pattern can be modified anytime by computing a new pattern with the on-board microcontroller (only ramps are allowed), or by downloading a new pattern from an external host computer, using the on-board RS232 port. In the latter case, any voltage shape complying restrictions mentioned above can be performed. These features open new paths to experimentation with new voltage shapes. As an added value, the system is able to store the real output voltage, which can be downloaded back to the host computer for analysis (study of the dielectric breakdown). This makes unnecessary the presence of additional elements, e.g. oscilloscopes. In this paper we present designs (including electronic schemes) for the system as well as measurement results obtained for different experimental samples and voltage patterns. The only important limitation of the device here described is the maximum frequency or maximum speed with which it can work. In the design carried out ramps with slopes higher than $400 \mathrm{~V} / \mathrm{ms}$ cannot be generated. For a more detailed analysis we are going to study each block of the schematic drawing shown in Fig. 1.

\section{AC-DC CONVERTER}

This stage is made up by an isolated transformer, followed by a diode bridge rectifier plus a filter capacitor. The value of this capacitor is basic to reduce to the maximum the voltage ripple with $50 \mathrm{~Hz}$, the peak-peak ripple in the output voltage of this stage is given by [2]

$$
V r \approx \frac{I_{d}}{2 f C}
$$

where $V r$ is the voltage ripple, $I_{\mathrm{d}}$ the mean current which supplies the diode bridge, $f$ the frequency of the electric network and $C$ the capacitance of the output capacitor.

According to equation 1 the ideal is to increase the filter capacitor to the maximum to reduce the ripple with $50 \mathrm{~Hz}$ to the maximum. Naturally this ripple goes to the DC-DC converter and we shall have it at the output Vo. Another possibility is to use an L-C filter with which a greater decrease of the voltage ripple is obtained.

\section{DC-DC CONVERTER}

The designed function generator is based on the principle of the switching power supplies. This type of power supplies has been used since the seventies, although it is in the eighties when it became popular in the electronic industry [2].

These systems are based on DC-DC switch-mode converters, with which transformers of all types can be obtained with quite an acceptable level of effectiveness. There are numerous references to this type of devices, explaining its design, its characteristics and the advantages they contribute with regard to other classic systems $[2,4]$. In principle the basic objective of this kind of converters is to obtain constant output voltages or at the most to be able to control the output voltage by the user. However, for our purposes the output must vary according to a pattern, which means a new way of using this type of devices. 
There are different types of switching power supplies. We have based the making of our device on a Flyback converter which in turn is just a derivation of the BuckBoost converters [2]. With regard to the construction of this stage a pulses transformer (high bandwidth), a fast switching power diode and a capacitor with a breakdown voltage of $1000 \mathrm{~V}$ have been used. On the output there are two voltage dividers made with power resistances.

A feature to bear in mind in these switching devices is the output voltage ripple, which is superimposed on that of the electric network. It is given by the following expression [2]:

$$
V r s=\frac{D T_{S}}{R_{S} C_{S}}
$$

where $V r s$ is the voltage ripple assuming the ripple of the stage AC-DC as void, $T_{S}$ is the switching time period of PWM, $D$ is the duty ratio $\left(T_{o n} / T_{S}\right), R_{S}$ is the load resistance on the output of the switching power supply and $C_{S}$ is the capacitance of the output capacitor.

To reduce the ripple it is necessary to increase the output resistance and the capacitance of the output capacitor. The increase of the latter clearly harms the speed with which the system responds, limiting the maximum slope that can be obtained in a ramp. Several values have been tested to try to keep the compromise between the decrease of the ripple and to be able to obtain higher slopes. In the end a relatively low value has been chosen, if the user wants to reduce the ripple he can always put on the output a shunt capacitor, with which the total capacitance would increase.

Another important feature for the construction of our function generator is the way the output voltage depends on the value of PWM [5]. For the Flyback dcdc converter such relation can be simplified depending on the operating model. This one can be: ContinuousConduction mode or Discontinuous-Conduction mode. For the continuous-conduction mode we may obtain [2]:

$$
V o=V d \frac{N 2}{N 1} \frac{D}{1-D}
$$

For the Discontinuous-Conduction mode [2]:

$$
\begin{gathered}
I_{o b, \max }=\frac{T_{S} V o}{2 L} \\
V o=D \frac{V d}{\sqrt{\frac{I o}{I_{o b, \max }}}}
\end{gathered}
$$

where $V o$ is the output voltage of the DC-DC converter, $N 2 / N 1$ is the transformer winding turns ratio, $V d$ is the input voltage of the DC-DC converter, $I o$ is the output intensity of the DC-DC converter and $L$ is the magnetizing inductance of the transformer. The use of one or other mode depends on the load, if the output intensity Io observes $I o<I_{o b, \max }(1-D)^{2}$ then the current conduction is discontinuous.

It may be observed that once the input and output voltage is known it is easy to tabulate which the value of PWM should be for a fixed load. It must be considered here that the first voltage divider (R2/R.3), which in principle is useful for the feedback of the output voltage, is also useful for load in open circuit. It is evident that the voltage on the output would tend to infinity without this load, with which a breakdown would be caused. On the other hand while a breakdown in the thin film is not produced the load on the output is the suitable of this voltage divider (R2/R3). When the breakdown is produced the output impedance changes sharply, which causes a response from the feedback system.

The second voltage divider is made up by a high impedance resistance (R4) which is useful for a current limiter and by another one with a very low impedance (R5), which is used as a sensor resistor. The specimen (the thin film) is inserted between these two resistances. No current is detected by the sensor resistor while breakdown does not occur and the load that the system has is due to the first voltage divider (R2/R3). When breakdown occurs shunt resistance (R4/R5) operate and decrease output impedance.

The waveform generated is completely distorted when breakdown occurs. If a ramp is generated the slope changes and the feedback factor can cause certain oscillations before recovering the slope, but all these effects are not significant since our objective is to detect the first breakdown. In fact we are not interested in what occurs after the first breakdown (although successive breakdowns can be produced and can be very interesting to be studied).

To feed the output voltage Vo and detect the current due to breakdown two voltage controlled oscillators (VCO) are used. The output of these devices are isolated by means of opto-isolators. Next they are connected to two timers microcontrollers, which are increased in each rise edge of the signal generated by VCO. At every sampling period such timers are read, reaching zero immediately. The values read indicate mean voltages in the sensor resistor between two sampling periods. 


\section{PWM GENERATOR}

The PWM generator is based on a classic design of counters, for which two PLD 22V10-5ns have been used. The two main features of every PWM digital generator are the frequency and the resolution. The PWM generator output is done by means of an optoisolator, which drives the driving stage power MOS. As a safety measure a feedback has been prepared through a sensor resistor connected to such transistor source and an internal reference voltage comparator and with an AND circuit the PWM is cut off when the threshold current is exceeded. This system is worked out to limit the maximum intensity on the Power MOS and it works when the safety system turnouts are exceeded, otherwise it has no effect on the system. The used MOS transistor is of the type $\mathrm{N}$ and it can support up to $800 \mathrm{~V}$ and $15 \mathrm{~A}$. Besides, it can convert into fast switching with the adequate gate drive.

For the correct operation of this stage it is necessary a force-ventilated coming from a ventilator which activates only when the system is generating a signal on its output.

\section{COMPUTER}

The first to be observed in the design is that the computer is electrically isolated from the rest of the system. It can be observed that there are three grounds in the system (GND1, GND2 and GND3). The computer in our case is a Siemens microcontroller that has evolved from the classic Intel 8051. The microcontroller functions are three :

- Voltage patterns calculation: the microcontroller obtains from the user the ramp slope and a desired voltage value for each sampling period. These voltage values are memorised in an array and represent the desired ramp. From this array and using a table the PWM value can be obtained for every sampling period. Our function generator can work in two ways: independently or connected to a PC. In the last case, any waveform can be stored in the memory. While these functions are carried out, the output voltage is zero and the microcontroller is working out. Once the necessary data to generate the waveform are stored in the memory, it informs the user by means of a LED diode.

- Interface with the user : the interface with the user is formed by an LCD display, a keyboard, three LED diodes, a trigger button and another one for reset. The system can communicate by means of RS232 to a PC. The PC can control the function generator by means of some commands. Arbitrary waveforms can even be downloaded in the microcontroller memory. In the first case, i.e., without the PC host, ramp data are introduced by means of the keyboard and LCD display, using a menu. In this case only output ramps can be obtained. While these functions are done by the microcontroller the output voltage is zero.
- Waveform generation and its control: when the user presses the trigger button (or the required command is sent by the PC via RS232) the output is activated. The microcontroller uses a timer to generate a periodical interruption whose period is a sampling period $(0.1 \mathrm{~ms}$ in our case). The real output voltage is read by means of the feedback circuit, new voltage and PWM values are loaded from the memory. Finally a PWM value is calculated as explained before. To sum up, the device process is developed in three stages : 1) data collection 2) to calculate the waveform and 3) to generate output signal when a proportional control with a feed-forward loop is used [3].

\section{EXPERIMENTAL RESULTS}

In Fig. 2 we show the current variation as well as the applied voltage ramp with a constant slope of $20 \mathrm{~V} / \mathrm{s}$ at a particular Au contact for a IBICVD ( Ion Beam Induced Chemical Vapour Deposition) sample. This voltage was applied separately to Au contacts of diameter $1 \mathrm{~mm}$ evaporated on the sample surface. From these two curves we can obtain the values of breakdown voltage, $\mathrm{V}_{\mathrm{BD}}$, and by numerical integration the charge to breakdown, $\mathrm{Q}_{\mathrm{BD}}$, or amount of charge that has to be transported before breakdown occurs. As we know the dielectric film thickness, the breakdown electric field, $E_{B D}$, is obtained. From the graph registered in the computer memory, the time that passes until the breakdown, TDDB, time dependent dielectric breakdown, can also be known. All the parameters that can be determined, $\mathrm{V}_{\mathrm{BD}}, \mathrm{E}_{\mathrm{BD}}, \mathrm{Q}_{\mathrm{BD}}$, TDDB, are fundamental to characterise the material and dielectric lifetime prediction.

We must do plenty of experiments for the determination of the previously pointed parameters to subsequently apply a particular suited distribution, namely extreme value statistics would be used to calculate the intrinsic breakdown and charge to breakdown of the sample [1]. With our equipment we can also apply a second technique which consists of the application of a constant voltage and measuring the time to breakdown, TDDB. As it is well known there is a relation between instantaneous or field breakdown and TDDB. This relation between the two kinds of experiments comes from the concept of $\mathrm{Q}_{\mathrm{DB}}$. In both techniques the breakdown occurs when the value of $\mathrm{Q}_{\mathrm{DB}}$ has been reached [1]. 


\section{REFERENCES}

[1] Breakdown: A review of oxide breakdown. Microelectronics Journal 27 (1996), 611-622.

[2] N. Mohan, T.M. Undeland, W.P. Robbins, Power Electronics: Converters, Applications, and Design. John Wiley \& Sons, NY 1989.

[1] J.F. Verweij, J.H. Klootwijk, Dielectric
[3] K. Ogata. Discrete-Time Control Systerns, Prentice-Hall, New Jersey, 1987.

[4] G. C. Chryssis. High Frequency Switching Power Supplies. Mc Graw Hill, 1984.

[5] V. Quercioli. Pulse Width Modulated (PWM) Power Supplies. Elsevier, 1993.

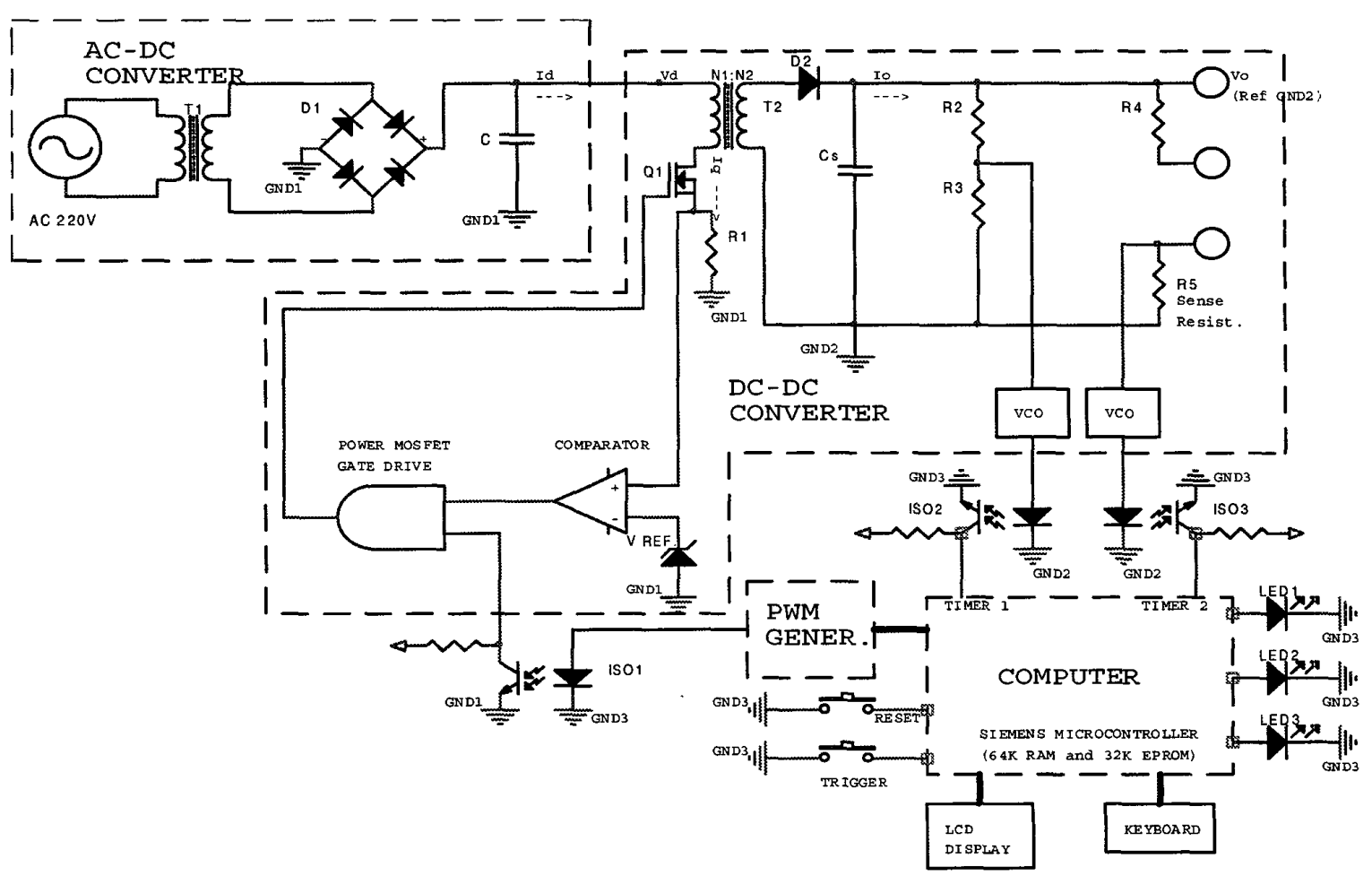

Figure 1. Function generator block diagram.

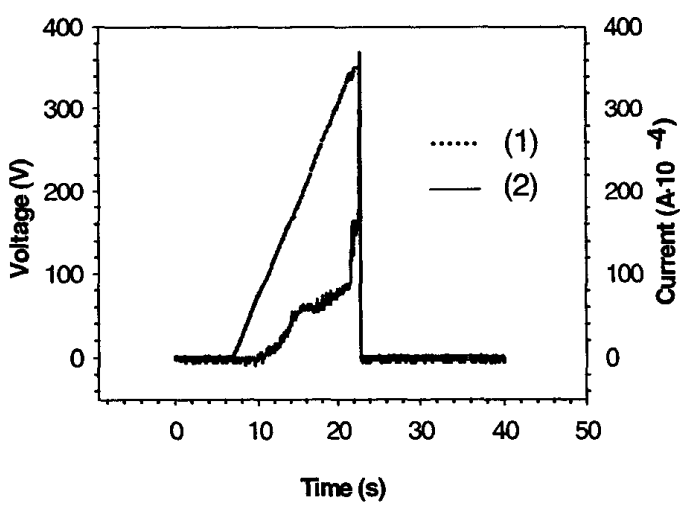

Figure 2. Electric signals from a particular Au contact in a IBICVD sample: (1) applied voltage ramp; (2) current variation. 